
\title{
ZS Research Square \\ Nanoparticle Synthesis from Biowaste and Its Potential as an Antimicrobial Agent
}

\author{
Rishana Saffar \\ Athira PV \\ Mount Carmel College (Autonomous) \\ Kangkana Kalita \\ Mount Carmel College (Autonomous) \\ Suba G A Manuel ( $\nabla$ subamanuel@mccblr.edu.in ) \\ Mount Carmel College (Autonomous) \\ Pradeep N \\ Mount Carmel College (Autonomous)
}

Mount Carmel College (Autonomous) https://orcid.org/0000-0002-9815-175X

\section{Research Article}

Keywords: Biowaste, silver nanoparticles, zinc nanoparticles, Punica granatum, Musa acuminata, Escherichia coli, Bacillus subtilis.

Posted Date: July 16th, 2021

DOl: https://doi.org/10.21203/rs.3.rs-718715/v1

License: (c) (i) This work is licensed under a Creative Commons Attribution 4.0 International License. Read Full License 


\section{Abstract}

Biosynthesized nanoparticles are gaining more importance because of their unique biological applications and can be synthesised using biowastes like fruit peels that acts as a better alternative against conventional physical and chemical methods. This work reports an ecofriendly, reliable and costeffective synthesis of silver and zinc oxide nanoparticles (Ag and ZnO NPs) chemically and biologically using fresh peel extracts of Punica granatum and Musa acuminata. The synthesised nanoparticles were characterized using UV-Visible spectroscopy, X-ray Diffraction (XRD), Scanning electron microscopy (SEM) and Energy dispersive X-ray analysis (EDS). The size of chemically and biosynthesized $\mathrm{Ag}$ and $\mathrm{ZnO}$ NPs ranged around 12-20 nm and 0.4-24 nm respectively. Both biosynthesized Ag and ZnO NPs were found to be nanospheres whereas chemically synthesised ZnO NPs were nanoflakes and showed maximum absorbance in the range of 250-550nm. Antimicrobial properties of the synthesized nanoparticles were assessed by well diffusion method against Gram negative Escherichia coli and Grampositive Bacillus subtilis. Biosynthesized Ag NPs were found to be more efficient against $E$. coli as compared to ZnO NPs wherein biosynthesized M. acuminata Ag NPs exhibiting maximum zone of inhibition $(28.4 \pm 14.66 \mathrm{~mm})$. Similarly, biosynthesized $\mathrm{ZnO}$ NPs were more efficient against $B$. subtilis as compared to biosynthesized Ag NPs wherein biosynthesized $P$. granatum ZnO NPs exhibiting maximum zone of inhibition $(14.08 \pm 3.03 \mathrm{~mm})$. Hence biosynthesized NPs can be exploited as a potential candidate for antimicrobial agents. Scientific and systematic use of biowaste for NPs synthesis could in turn result in a sustainable solution for waste management.

\section{Introduction}

Waste generation and disposal are one of the significant problems persisting in India. Urban India generates 62 million tonnes of municipal solid waste each year, and around $80 \%$ of this is dumped in the landfill every day ${ }^{1}$. The generation of bio-wastes has accelerated due to the increased population growth, food production, urbanisation, and industrialisation. In developing countries like India, these wield tremendous impacts on soil health, climate change, and overall environment ${ }^{2}$. Hence scientific and systematic use of biowaste is necessary in order to eliminate tremendous heap of biowaste as well as to gain maximum benefit out of it.

Nanotechnology comprises nanoscale science, that deals with nanoparticles, their properties, synthesis, characterisation, and application in various fields. The use of bio-wastes for the synthesis of nanoparticles that do not produce toxic wastes during their synthesis is considered safe and ecologically sound for nanomaterial fabrication as an alternative to conventional physical and chemical methods ${ }^{3}$.

The usage of agro-wastes from fruits has acquired significant attention in recent years due to their availability and cost-effectiveness. Punica granatum fruit peel had well-acknowledged for containing a high number of phenolic compounds as sources of natural antioxidants. These compounds play a significant role in the synthesis of nanoparticles via green synthesis. Musa acuminata are also rich in polymers such as lignin, cellulose, hemicellulose, and pectin have also used in the synthesis of various 
nanoparticles $4,5,6,7$. The redox properties of the phenolic compounds are responsible for the antioxidant activity and play a vital role in absorbing and neutralizing free radicals, quenching singlet and triplet oxygen or decomposing peroxides ${ }^{8}$.

The silver nanoparticles (Ag NPs) show efficient antimicrobial property when compared to other salts as they have an extensive surface area. Hence it provides better contact with microorganisms and also penetrates inside the organism and preferably attack the respiratory chain, cell division that is eventually leading to cell death.

Zinc oxide nanoparticles (ZnO NPs) act as an antibacterial material because of its unique properties, like high specific surface area and high activity to block a broad scope of pathogenic agents. Reports had suggested that the antibacterial toxicity mechanisms of ZnO NPs were due to their ability to induce excess Reactive Oxygen Species (ROS) generation, such as superoxide anion, hydroxyl radicals, and hydrogen peroxide production ${ }^{9}$.

In the present study, efforts have made to synthesise nanoparticles from the waste fruit peels of Punica granatum and Musa acuminata. Silver and zinc oxide nanoparticles have synthesised both using fresh peel extract and powder preparation using the peels and they were also chemically synthesised. Characterisation of nanoparticles was done using UV-visible spectroscopy, $x$-ray diffraction analysis, and scanning electron microscopy. A comparative evaluation of the antimicrobial properties of fruit peel extract, chemically synthesised nanoparticles, and nanoparticles synthesised from peels had done to affirm its efficiency.

\section{Related works}

Over the past decade, biosynthesis of nanoparticles from waste is gaining much importance. Collagen, chitin and chitosan, hydroxyapatites and bio-silica/silicates had used for the construction of bioplastics and nanocarbon structure which acts as a support for metal nanoparticles ${ }^{10}$. Fruit waste and plant waste are commonly used for biosynthesis of nanoparticles as these are readily available, economical, nontoxic and compatible with the environment ${ }^{11}$. Banana peel extract is a natural reducing agent and also rich in polymers like lignin, cellulose, hemicellulose and pectin and thus used for the synthesis of silver nanoparticles ${ }^{6}$. Banana fruit waste extract contains biomolecules which act as reducing, stabilizing and capping agents and utilized for the synthesis of gold nanoparticles ${ }^{12}$. Pomegranate peel extract comprised of phenolic compounds, gallic acid and other fatty acids, flavonols, flavones, flavanones, and anthocyanidins and their reducing property had exploited in the production of silver and gold nanoparticles. Egg shells and shrimp peels that are thrown away as waste had used for the synthesis of various nanoparticles ${ }^{3}$. Synthesis from plant extract is accepted over other biological processes as it is relatively simple, readily available and does not require the maintenance of cell cultures ${ }^{12}$.

\section{Materials And Methods}


Chemical synthesis of Ag NPs and ZnO NPs: Aqueous silver nitrate solution (0.003M) was continuously stirred for about 15 minutes at $80^{\circ} \mathrm{C}$. Aqueous trisodium citrate solution $(0.03 \mathrm{M})$ was added slowly until the clear solution turns into golden yellow, indicating the formation of $\mathrm{Ag} \mathrm{NPs}^{13}$. ZnO NPs were synthesized by the wet chemical method using zinc nitrate and sodium hydroxide as precursors. An aqueous solution of zinc nitrate $(0.1 \mathrm{M})$ was kept under constant stirring to dissolve the zinc nitrate, and an aqueous solution of sodium hydroxide ( $0.8 \mathrm{M})$ was also prepared in the same way. After the complete dissolution of zinc nitrate, under high-speed constant stirring aqueous sodium hydroxide solution was added drop by drop touching the walls of the beaker. The reaction proceeded for $2 \mathrm{~h}$ and the solution was allowed to settle overnight. The precipitated ZnO NPs were cleaned three times with deionised water and ethanol to remove the by-products which were bound with the NPs and then dried at $60^{\circ} \mathrm{C}$. White powder formation, indicated the formation of the NPs ${ }^{14}$.

Spectral analysis for the development of NPs was observed by UV-Visible spectroscopy (Shimadzu, UV 1700 PharmaSpec, scanning range: $250-550 \mathrm{~nm}$ ). Further characterization was done by scanning electron microscope and X-Ray Diffraction Analysis ${ }^{13,14}$.

Biosynthesis of Ag NPs: Peels of $P$. granatum $(50 \mathrm{~g})$ washed and taken in Erlenmeyer flask and then boiled with ultrapure water $(250 \mathrm{~mL})$ for $10-15$ minutes. Contents were filtered using Whatman filter paper (no. 1) to prepare the aqueous fruit peel extract, which was stored for further use. An aqueous solution of silver nitrate $(1 \mathrm{mM})$ was prepared and to this fruit peel extract $(1.8 \mathrm{~mL})$ was added and incubated at room temperature for $5 \mathrm{~min}$. The formation of brown-yellow colour indicated the formation of NPs ${ }^{8}$.

M. acuminata peels were washed, dried at room temperature, and sliced into small pieces. Peels (100g) were weighed and boiled in distilled water $(100 \mathrm{~mL})$ for 30 minutes at $90^{\circ} \mathrm{C}$. Contents were homogenised, and filtered using Whatman filter paper (no. 1). This filtrate was treated with an equal volume of chilled acetone, and the resultant precipitate was resuspended in distilled water and stored at $4^{\circ} \mathrm{C}$ for further use. An aqueous $1 \mathrm{mM}$ solution of silver nitrate solution was prepared. To this peel extract $(1 \mathrm{~mL})$ was added and to avoid photoactivation incubated in the dark at $30^{\circ} \mathrm{C}$. Yellowish-brown to light reddish-brown colour indicated the formation of the NPs ${ }^{15}$.

Biosynthesis of ZnO NPs: P. granatum fruit peels were washed thoroughly with tap water and distilled water and dried in the oven at $45^{\circ} \mathrm{C}$ and ground using an electric blender into a fine powder and stored at room temperature for further use. For the preparation of the extract, the peel powder was extracted in distilled water at $65^{\circ} \mathrm{C}$ for 60 minutes in the ratio of $1: 10$. The extract was then filtered using mesh to remove large particles and centrifuged at $10,000 \mathrm{rpm}$ for 10 minutes and kept at $4^{\circ} \mathrm{C}$ for further experiments Sol-gel method was used to synthesize $P$. granatum ZnO NPs. To the fruit peel powder extract, zinc nitrate hexahydrate was added under vigorous stirring at $90^{\circ} \mathrm{C}$ in the ratio $1: 10$. The solution was stirred until the aqueous solvent was removed, leaving a gel-like product indicating the formation of the NPs ${ }^{4}$. 
Dried fine peel $(50 \mathrm{~g})$ of $M$. acuminata was weighed and boiled with sterile distilled water ( 1 litre) for 20 minutes at $60^{\circ} \mathrm{C}$. It was allowed to cool to room temperature and was filtered using Whatman filter paper (no.1). The extract was stored in the refrigerator for further experiments. For the synthesis of ZnO NPs, $500 \mathrm{~mL}$ of peel powder extract was taken in a clean conical flask. To this zinc nitrate $(10 \mathrm{~g})$ was added and mixed thoroughly and kept in shaker incubator for 2 hours at $150 \mathrm{rpm}$. After incubation, the mixture was allowed to cool down to room temperature ${ }^{16}$.

\section{Characterisation of Nanoparticles}

UV-Visible spectroscopy: The optical properties of ZnO and Ag NPs obtained through chemical synthesis and biosynthesis was charaterized using UV-Vis spectroscopy. The medium was prepared by diluting small aliquots of the reaction mixture with distilled water and transferred to the cuvette, and analysis was done using a UV-Vis spectrophotometer (Shimadzu UV-Visible Spectrophotometer). The absorption range for both ZnO and Ag NPs were scanned from 250-550nm ${ }^{17}$.

XRD analysis: The dried powdered sample was used to perform XRD (Rigaku Smartlab X-Ray Diffractometer) for confirming the nature and size of ZnO and Ag NPs (CeNSE lab IISc, Bangalore) ${ }^{18}$. By using Scherrer's equation, the average crystallite size was determined.

SEM and Energy Dispersive X-RAY spectroscopy analysis: The SEM and EDX (Zeiss, Ultra 55 Field Emission Scanning Electron Microscope) analysis had used to determine the surface structure and to determine the chemical purity, elemental composition, and stoichiometry of chemically and biosynthesized Ag NPs and ZnO NPs (CeNSE lab IISc, Bangalore) ${ }^{18}$.

Antimicrobial assay by well diffusion method: Pure cultures of $E$. coli and B. subtilis were cultured on Mueller- Hinton (MH) agar slants and incubated at $37^{\circ} \mathrm{C}$. Loopful of culture was suspended in $\mathrm{MH}$ broth and incubated overnight at $37^{\circ} \mathrm{C}$. Sterile Petri plates were poured with $\mathrm{MH}$ agar aseptically and allowed to solidify. The incubated bacterial culture was swabbed uniformly. Samples $(40 \mu \mathrm{L})$ were poured into each well, which was incubated at $37^{\circ} \mathrm{C}$ for $18 \mathrm{~h}$. After incubation, the zone of inhibition was measured. Distilled water was used as a negative control. A comparison was made between the chemically synthesized NPs, fruit peel extracts and biosynthesized NPs ${ }^{19}$.

\section{Results And Discussion}

Synthesised NPs: The formation of chemically synthesised Ag NPs was indicated by the clear solution turning into golden yellow which is in accordance with the previous literature ${ }^{13}$. The formation of biosynthesised Ag NPs from P. granatum and $M$. acuminata fruit peels was indicated by the formation of a brown-yellow solution in accordance with the literature ${ }^{5,8}$. Chemically synthesised ZnO NPs was indicated by the formation of white powder ${ }^{14}$. Biosynthesised ZnO NPs from $P$. granatum fruit peels were indicated by the formation of a gel-like product ${ }^{4}$. The formation of biosynthesised $\mathrm{ZnO}$ NPs from $M$. acuminata fruit peels were indicated by the formation of brown colour solution. 


\section{Characterization of NPs}

UV-visible spectral analysis of Ag and ZnO NPs: The optical properties of chemically and biosynthesised Ag and ZnO NPs were characterized using UV-Visible spectrophotometer. The absorption maxima for chemically synthesised Ag NPs were observed around 422nm which correlates with previous literature in which absorption maxima was observed around $430 \mathrm{~nm}^{20}$. The absorption maxima for biosynthesised $\mathrm{Ag}$ NPs from $P$. granatum were observed around $371 \mathrm{~nm}^{21}$. The absorption maxima for biosynthesised Ag NPs from M. acuminata were observed around $438 \mathrm{~nm}$ which correlates with earlier finding in which it was observed at the spectrum range of 426.50 to $452.00 \mathrm{~nm}^{15}$.

Previous literature states that ZnO NPs show absorption maxima in a spectrum range of $360-380 \mathrm{~nm}^{22}$. Chemically synthesised $\mathrm{ZnO}$ showed maximum absorption at $363 \mathrm{~nm}$ which correlates with the spectral range of $\mathrm{ZnO}$, thus confirming the formation of ZnO NPs. Biosynthesised ZnO NPs from $P$. granatum showed maximum absorbance around $364 \mathrm{~nm}^{4}$. Biosynthesised ZnO NPs from $M$. acuminata showed maximum absorbance around $313 \mathrm{~nm}$, which correlates with the previous literature which was observed around $351 \mathrm{~nm}^{16}$. The chemically synthesised and biosynthesised ZnO NPs exhibited a similar range of absorption maxima. Thus, the absorption range for Ag and ZnO NPs was observed between 250-550 nm thus confirming the formation of respective NPs.

X-Ray diffraction (XRD) analysis of the synthesised NPs: XRD analysis was done using Rigaku X-Ray Diffractometer to determine the crystallite size, and lattice strain of the synthesised Ag and ZnO NPs. The XRD graphs obtained was compared with the respective standard XRD graphs of Ag and ZnO NPs to confirm the presence of NPs (Figure 1(a), (b), (c), (d), (e), (f)). Scherrer's equation was used to calculate the average crystallite size and lattice strain (Table 1).

The various diffraction peak intensities are a result of the polycrystalline nature of the NPs. Previous literature states that the intense reflection at (111 peak) compared to other peaks indicates the growth direction of Ag nanocrystals (Figure 1(a), (b) \&(c)). When compared to other peaks, 101 peak intensity was the strongest for chemically synthesised ZnO NPs (Figure1(d)). The peak intensity was the strongest at (100) as compared to other peaks in both $P$. granatum and $M$. acuminata biosynthesized ZnO NPs (Figure 1(e), (f)). Due to various size-dependent factors, irregular peak positions, heights and widths are obtained ${ }^{13}$. Impurities present in the sample gives peaks other than the Ag characteristic peaks ${ }^{14}$ and $\mathrm{ZnO}$ characteristic peaks.

From the XRD analysis done it can be concluded that the size of chemically and biosynthesized Ag NPs were estimated to range around 12 to $20 \mathrm{~nm}$ and the size of chemically and biosynthesized ZnO NPs were estimated to range around $0.4-24 \mathrm{~nm}$.

Characterization using scanning electron microscope (SEM): SEM analysis was done using Zeiss, Ultra 55 Field Emission Scanning Electron Microscope to determine the surface structure of the Ag NPs and 
ZnO NPs drop-cast sample. SEM images indicated the length $(\mathrm{nm})$ and shape of the synthesised nanoparticles (Table 2).

The SEM images of the chemically synthesised Ag drop-cast sample were nanospheres (Figure 2 (a)), whereas the SEM images of the chemically synthesised ZnO drop cast sample (Figure 2 (g)) were nanoflakes. SEM images of both biosynthesised Ag and ZnO NPs were nanospheres (Figure 2 (c), (e), (i), (k)). SEM images of P.granatum NPs is similar to previous literature ${ }^{23}$. SEM analysis of synthesised NPs indicated they are spherical and their sizes were in the range of 30-63 nm.

EDX analysis gives qualitative as well as the quantitative status of elements that may be involved in the formation of nanoparticles (Table $3 \& 4$ ). EDX analysis had also carried out to determine the chemical purity, elemental composition, and stoichiometry of the synthesised NPs ${ }^{18}$. The elemental profile of synthesised Ag NPs using BPE shows a higher count of silver, confirming the NPs formation ${ }^{5}$.

The elemental profile of Ag NPs from P. granatum and chemically synthesised also showed higher count of Ag, confirming the NPs formation. Similarly, EDX analysis of chemically and biosynthesised ZnO NPs showed higher count of zinc oxide, even though other elements like zinc and sodium were present, which confirms the formation of $\mathrm{ZnO}$ NPs.

\section{Antimicrobial activity of synthesised NPs:}

The antimicrobial assay was carried out by the well diffusion method against different human pathogenic Gram-positive and Gram-negative bacterial species (E. coli and B. subtilis). Both chemically and biosynthesized silver and zinc oxide nanoparticles were used to assess their antimicrobial activity.

The biologically synthesized nanoparticle had found to be more efficient than the chemically synthesized nanoparticle for antimicrobial activity. The maximum zone of inhibition was observed in $M$. acuminata Ag NPs $(28.4 \pm 14.66 \mathrm{~mm})$ against $E$. coli compared to chemically synthesized silver NPs $(16.8 \pm 8.44 \mathrm{~mm})$. Also, $M$. acuminata Ag NPs showed a higher zone of inhibition as compared to $P$. granatum Ag NPs $(20 \pm 12.17 \mathrm{~mm})$ against E. coli.

M. acuminata ZnO NPs $(10.4 \pm 0.89 \mathrm{~mm})$ and $P$. granatum ZnO NPs $(12.8 \pm 1.79 \mathrm{~mm})$ exhibited higher zone of inhibition as compared to chemically synthesized ZnO NPs $(6.4 \pm 3.29 \mathrm{~mm})$ against $E$. coli wherein P. granatum ZnO NPs showed a higher zone of inhibition as compared to $M$. acuminata ZnO NPs. Biosynthesized Ag NPs are more efficient as an antimicrobial agent as than biosynthesized ZnO NPs against E. coli. Hence it is concluded that biosynthesized NPs are more efficient as compared to chemically synthesized NPs against $E$. coli.

The antimicrobial properties of chemically and biosynthesised silver and zinc oxide NPs against $E$. coli had analysed by conducting Student t-test. A null hypothesis and an alternative hypothesis were proposed. The null hypothesis proposed states that there is no significant difference between the two groups and the alternative hypothesis states that there is a significant difference between the groups. The 
p-value for the test calculated as 0.0275 and found that the test is significant as the p-value obtained is less than 0.05. Hence the Null hypothesis was accepted (Table 5, Figure 3).

P. granatum peels used for the biosynthesis of silver nanoparticles showed inhibition against Staphylococcus aureus, Pseudomonas aeruginosa and Escherichia coli pathogens ${ }^{23}$. Biosynthesized Ag NPs had reported as more effective than chemically synthesized Ag NPs.

P. granatum Ag NPs showed high zone of inhibition as compared to M. acuminata Ag NPs, which showed the least zone of inhibition against $B$. subtilis. The antimicrobial activity of chemically synthesised $\mathrm{Ag}$ NPs $(9.2 \pm 2.28 \mathrm{~mm})$ was less efficient as compared to $P$. granatum Ag NPs $(11.2 \pm 2.68 \mathrm{~mm})$, and it was more efficient as compared to $M$. acuminata Ag NPs $(7.2 \pm 3.63 \mathrm{~mm})$ against $B$. subtilis. Chemically synthesized ZnO NPs $(6.4 \pm 3.29 \mathrm{~mm})$ showed the least zone of inhibition as compared to $M$. acuminata ZnO NPs $(10.4 \pm 0.89 \mathrm{~mm})$ and $P$. granatum ZnO NPs $(14.8 \pm 3.03 \mathrm{~mm})$ whereas $P$. granatum ZnO NPs showed a higher zone of inhibition as compared to M. acuminata ZnO NPs. Biosynthesized ZnO NPs are more efficient as an antimicrobial agent as compared to biosynthesized Ag NPs against $B$. subtilis. Hence it is concluded that biosynthesized NPs are more efficient as compared to chemically synthesized NPs against $B$. subtilis.

The antimicrobial properties of chemically and biologically synthesized silver and zinc oxide against $B$. subtilis had analyzed conducting Student t-test. A null hypothesis and an alternative hypothesis had proposed. The null hypothesis proposed states that there is no significant difference between the two groups and the alternative hypothesis states that there is a significant difference between the groups. The p-value for the test calculated as 0.327 and found that the test is non-significant as the p-value obtained is more than 0.05 . Hence the alternative hypothesis was accepted (Table 6, Figure 4).

The use of Musa peels for the synthesis of ZnO NPs were compared with commercial bulk ZnO particles and also with ZnO NPs prepared using nonionic and anionic surfactants ${ }^{24}$. It was found that the $\mathrm{ZnO}$ NPs films prepared from the Musa peels exhibited DPPH scavenging activity and was higher than that of the commercial film. Apart from this, the films with ZnO NPs exhibited antibacterial properties towards $S$. aureus and E. coli. chitosan/zinc oxide nanocomposites were tested against Gram-positive and Gramnegative bacteria and the results showed that the nanocomposite possesses thermal and antibacterial properties that got improved with the presence of ZnO NPs when compared with chitosan alone ${ }^{25}$.

Comparative analysis of antimicrobial activities of biosynthesized Ag NPs and ZnO NPs showed that biosynthesized Ag NPs were more efficient against $E$. coli as compared to biosynthesized ZnO NPs. Similarly biosynthesized ZnO NPs were more efficient against $B$. subtilis as compared to biosynthesized Ag NPs.

\section{Conclusion}

Biological methods for the synthesis of nanoparticles employ the use of plants, fungus, bacteria, and algae. These methods had adopted to cut down the use of toxic chemicals which, in excessive quantities, 
are hazardous to the environment. The present study aims at sustainable utilization of waste fruit peels for the synthesis of nanoparticles. The synthesised NPs has the potential to serve as an efficient antimicrobial agent. These NPs also have a potential role in the fields of biology and biomedicine, environment, industries, food, and agriculture.

\section{Declarations}

\section{Acknowledgments}

We gratefully acknowledge the CeNSE lab members (Indian Institute of Science, Bengaluru) for helping us with XRD and SEM facility. Kind thanks to Mr Pradeep, Mr Amit, Mr Abhishek, Ms Vanitha, Mr Arun Babu and Ms Suma for the guidance to conduct and analyse the XRD and SEM results.

\section{Code Availability}

Not applicable.

\section{Funding}

Not applicable.

\section{Ethics declarations}

Ethics Approval

Not applicable.

\section{Consent to Participate}

Not applicable.

\section{Consent for Publication}

The participants gave their consent for publication.

\section{Conflict of Interest}

The authors declare no competing interests

\section{References}

1. Down to Earth. (2019). India's challenges in waste management. Retrieved from https://www.downtoearth.org.in/blog/waste/india-s-challenges-in-waste-management-56753

2. Manna, M. C., Rahman, M. M., Naidu, R., Sahu, A., Bhattacharjya, S., Wanjari, R. H., Patra, A. K., Chaudhari, S. K., Majumdar, K. \& Khanna, S. S. (2018). Bio-waste management in subtropical soils of 
India: future challenges and opportunities in agriculture. Advances in Agronomy, 152, 87-148.

3. Mahgoub, S., \& Samaras, P. (2014, June). Nanoparticles from biowastes and microbes: Focus on role in water purification and food preservation. In 2nd International Conference on Sustainable Solid Waste Management.

4. Sukri, S. N. A. M., Shameli, K., Wong, M. M. T., Teow, S. Y., Chew, J., \& Ismail, N. A. (2019). Cytotoxicity and antibacterial activities of plant-mediated synthesized zinc oxide ( $\mathrm{ZnO})$ nanoparticles using Punica granatum (pomegranate) fruit peels extract. Journal of Molecular Structure, 1189, 57-65.

5. Ibrahim, H. M. (2015). Green synthesis and characterization of silver nanoparticles using banana peel extract and their antimicrobial activity against representative microorganisms. Journal of radiation research and applied sciences, 8(3), 265-275.

6. Narayanamma, A., Rani, A., \& Raju, M. E. (2016). Natural synthesis of silver nanoparticles by banana peel extract and as an antibacterial agent. Journal of Polymer and Textile Engineering, 3(1), 17-25.

7. Bankar, A., Joshi, B., Kumar, A. R., \& Zinjarde, S. (2010). Banana peel extract mediated novel route for the synthesis of silver nanoparticles. Colloids and Surfaces A: Physicochemical and Engineering Aspects, 368(1-3), 58-63.

8. Ahmad, N., \& Sharma, S. (2012). Biosynthesis of silver nanoparticles from biowaste pomegranate peels. International Journal of Nanoparticles, 5(3), 185-195.

9. Jiang, J., Pi, J., \& Cai, J. (2018). The advancing of zinc oxide nanoparticles for biomedical applications. Bioinorganic chemistry and applications, 2018.

10. Xu, C., Nasrollahzadeh, M., Selva, M., Issaabadi, Z., \& Luque, R. (2019). Waste-to-wealth: biowaste valorization into valuable bio (nano) materials. Chemical Society Reviews, 48(18), 4791-4822.

11. Kulkarni, N., \& Muddapur, U. (2014). Biosynthesis of metal nanoparticles: a review. Journal of Nanotechnology, 2014.

12. Kumar, I., Mondal, M., \& Sakthivel, N. (2019). Green synthesis of phytogenic nanoparticles. In Green synthesis, characterization and applications of nanoparticles (pp. 37-73). Elsevier.

13. Suriati, G., Mariatti, M., \& Azizan, A. (2014). Synthesis of silver nanoparticles by chemical reduction method: Effect of reducing agent and surfactant concentration. International Journal of Automotive and Mechanical Engineering, 10, 1920.

14. Talam, S., Karumuri, S. R., \& Gunnam, N. (2012). Synthesis, characterization, and spectroscopic properties of ZnO nanoparticles. International Scholarly Research Notices, 2012.

15. Suryanto, E., Taroreh, M. I., \& Momuat, L. I. (2019). Singlet Oxygen Quenching activity of Silver Nanoparticles Synthesized using Goroho Banana Peel (Musa acuminata). International Journal of ChemTech Research, 12(1), 258-268.

16. Ananthalakshmi, R., Rajarathinam, S. R.X., Lavanya, A., Sadiq, A. M., \& Gomathi, A. C. (2017). Synthesis and characterization of zinc oxide nanoparticles using aqueous extract of Banana Peel (Musa acuminata L.). International Journal of Basic and Applied Research, 7(12), 98-107 
17. Mourdikoudis, S., Pallares, R. M., \& Thanh, N. T. (2018). Characterization techniques for nanoparticles: comparison and complementarity upon studying nanoparticle properties. Nanoscale, 10(27), 12871-12934.

18. Chaudhuri, S. K., \& Malodia, L. (2017). Biosynthesis of zinc oxide nanoparticles using leaf extract of Calotropis gigantea: characterization and its evaluation on tree seedling growth in nursery stage. Applied Nanoscience, 7(8), 501-512.

19. Valsaraj, R., Pushpangadan, P., Smitt, U. W., Adsersen, A., \& Nyman, U. (1997). Antimicrobial screening of selected medicinal plants from India. Journal of Ethnopharmacology, 58(2), 75-83.

20. Gudikandula, K., \& Charya Maringanti, S. (2016). Synthesis of silver nanoparticles by chemical and biological methods and their antimicrobial properties. Journal of Experimental Nanoscience, 11(9), 714-721.

21. Devanesan, S., AlSalhi, M. S., Balaji, R. V., Ranjitsingh, A. J. A., Ahamed, A., Alfuraydi, A. A., AlQahtani, F.Y., Aleanizy, F.S. \& Othman, A. H. (2018). Antimicrobial and cytotoxicity effects of synthesized silver nanoparticles from Punica granatum peel extract. Nanoscale research letters, 13(1), 1-10.

22. Chieng, B. W., \& Loo, Y. Y. (2012). Synthesis of ZnO nanoparticles by modified polyol method. Materials Letters, 73, 78-82.

23. Shanmugavadivu, M., Kuppusamy, S., \& Ranjithkumar, R. (2014). Synthesis of pomegranate peel extract mediated silver nanoparticles and its antibacterial activity. Am J Adv Drug Deliv, 2(2), 174182.

24. Hamed, O. (2018). Fabrication of zinc oxide nanoparticles and films by banana peel extract food waste and investigation on their antioxidant and antibacterial activities.

25. Toiserkani, H. (2016). Fabrication and characterization chitosan/functionalized zinc oxide bionanocomposites and study of their antibacterial activity. Composite Interfaces, 23(3), 175-189.

\section{Tables}

Table 1: Crystallite size and lattice strain for the chemically and biosynthesised Ag and ZnO NPs

\begin{tabular}{|lll|}
\hline Sample & $\begin{array}{l}\text { Crystallite size } \\
(\mathrm{nm})\end{array}$ & Lattice strain \\
\hline Chemically synthesised Ag NPs & 19.41 & 0.006 \\
\hline Ag NPs using P. granatum peels & 13.88 & 0.008 \\
\hline Ag NPs using M. acuminata peels & 13.64 & 0.008 \\
\hline Chemically synthesised ZnO NPs & 24.419 & 0.005 \\
\hline ZnO NPs using P. granatum peels & 1.109 & 0.203 \\
\hline ZnO NPs using M. acuminata peels & 0.41 & 0.305 \\
\hline
\end{tabular}


Table 2: Length $(\mathrm{nm})$ and shape of the chemically and biosynthesised Ag and ZnO NPs

\begin{tabular}{|lll|}
\hline Sample & Length $(\mathrm{nm})$ & Shape \\
\hline Chemically synthesised Ag NPs & $58.59 ; 48.76 ; 48.32$ & Nanosphere \\
\hline Ag NPs from $P$. granatum peels & $53.78 ; 65.83 ; 70.55$ & Nanosphere \\
\hline Ag NPs from $M$. acuminata peels & $63.98 ; 58.59 ; 60.11$ & Nanosphere \\
\hline Chemically synthesized ZnO NPs & $63.38 ; 35.16 ; 59.75$ & Nanoflakes \\
\hline ZnO NPs from $P$. granatum peels & 4,$9010 ; 54,770$ & Nanosphere \\
\hline ZnO NPs from M. acuminata peels & $34.16 ; 43.94 ; 34.16$ & Nanosphere \\
\hline
\end{tabular}

Table 3: EDX analysis of Chemically and biosynthesised Ag NPs

\begin{tabular}{|c|c|c|c|c|c|c|}
\hline Element & Sample & & & & & \\
\hline \multirow[t]{2}{*}{ Name } & \multicolumn{2}{|c|}{$\begin{array}{l}\text { Chemically synthesised } \\
\text { AgNPs }\end{array}$} & \multicolumn{2}{|c|}{$\begin{array}{l}\text { Ag NPs from } P \\
\text { granatum peels }\end{array}$} & \multicolumn{2}{|c|}{$\begin{array}{l}\text { Ag NPs from } M \text {. } \\
\text { acuminata peels }\end{array}$} \\
\hline & Weight\% & Atomic\% & Weight\% & Atomic\% & Weight\% & Atomic\% \\
\hline Oxygen & 6.44 & 31.71 & 10.91 & 45.22 & 8.7 & 39.12 \\
\hline Silver & 93.56 & 68.29 & 89.09 & 54.78 & 91.3 & 60.88 \\
\hline
\end{tabular}

Table 4: EDX analysis of chemically and biosynthesised ZnO NPs

\begin{tabular}{|lllllll|}
\hline Element & \multicolumn{2}{l}{ Sample } & & & & \\
Name & \multicolumn{2}{l}{$\begin{array}{l}\text { Chemically synthesised } \\
\text { ZnO NPs }\end{array}$} & \multicolumn{2}{l|}{$\begin{array}{l}\text { ZnO NPs from } P . \\
\text { granatum peels }\end{array}$} & \multicolumn{2}{l|}{$\begin{array}{l}\text { ZnO NPs from } M \text {. } \\
\text { acuminata peels }\end{array}$} \\
\hline Weight\% & Atomic\% & Weight\% & Atomic\% & Weight\% & Atomic\% \\
\hline Oxygen & 23.21 & 49.07 & 55.48 & 79.67 & 13.54 & 33.65 \\
\hline Sodium & 65.07 & 33.67 & 37.08 & 13.1 & 74.2 & 45.14 \\
\hline
\end{tabular}

Table 5: In vitro analysis of the antimicrobial activity of $\mathrm{Ag}$ and $\mathrm{ZnO}$ NPs against $E$. coli. 


\begin{tabular}{|c|c|c|c|c|c|}
\hline \multicolumn{6}{|c|}{ Zone of Inhibition (mm) } \\
\hline Ag NPs & & & Zno NPs & & \\
\hline $\begin{array}{l}\text { Chemically } \\
\text { synthesized } \\
\text { NPs }\end{array}$ & $\begin{array}{l}\text { M. } \\
\text { acuminata NPs }\end{array}$ & $\begin{array}{l}P_{\text {granatum NPs }} \\
\text { gran }\end{array}$ & $\begin{array}{l}\text { Chemically } \\
\text { synthesized } \\
\text { NPs }\end{array}$ & $\begin{array}{l}\text { M. } \\
\text { acuminata NPs }\end{array}$ & $\begin{array}{l}\text { P. } \\
\text { granatum NPs }\end{array}$ \\
\hline $16.8 \pm 8.44$ & $28.4 \pm 14.66$ & $20 \pm 12.17$ & $6.4 \pm 3.29$ & $10.4 \pm 0.89$ & $12.8 \pm 1.79$ \\
\hline
\end{tabular}

Table 6: In vitro analysis of the antimicrobial activity of $\mathrm{Ag}$ and $\mathrm{ZnO}$ NPs against $B$. subtilis.

\begin{tabular}{|c|c|c|c|c|c|}
\hline \multicolumn{6}{|c|}{ Zone of Inhibition (mm) } \\
\hline Ag NPs & & & ZnO NPs & & \\
\hline $\begin{array}{l}\text { Chemically } \\
\text { synthesized } \\
\text { NPs }\end{array}$ & $\begin{array}{l}\text { M. } \\
\text { acuminata NPs }\end{array}$ & $\begin{array}{l}\text { P. } \\
\text { granatum NPs }\end{array}$ & $\begin{array}{l}\text { Chemically } \\
\text { synthesized } \\
\text { NPs }\end{array}$ & $\begin{array}{l}\text { M. } \\
\text { acuminata NPs }\end{array}$ & $\begin{array}{l}P_{\text {granatum NPs }} \\
\text { grats }\end{array}$ \\
\hline $9.2 \pm 2.28$ & $7.2 \pm 3.63$ & $11.2 \pm 2.68$ & $6.4 \pm 3.29$ & $10.4 \pm 0.89$ & $14.8 \pm 3.03$ \\
\hline
\end{tabular}

\section{Figures}



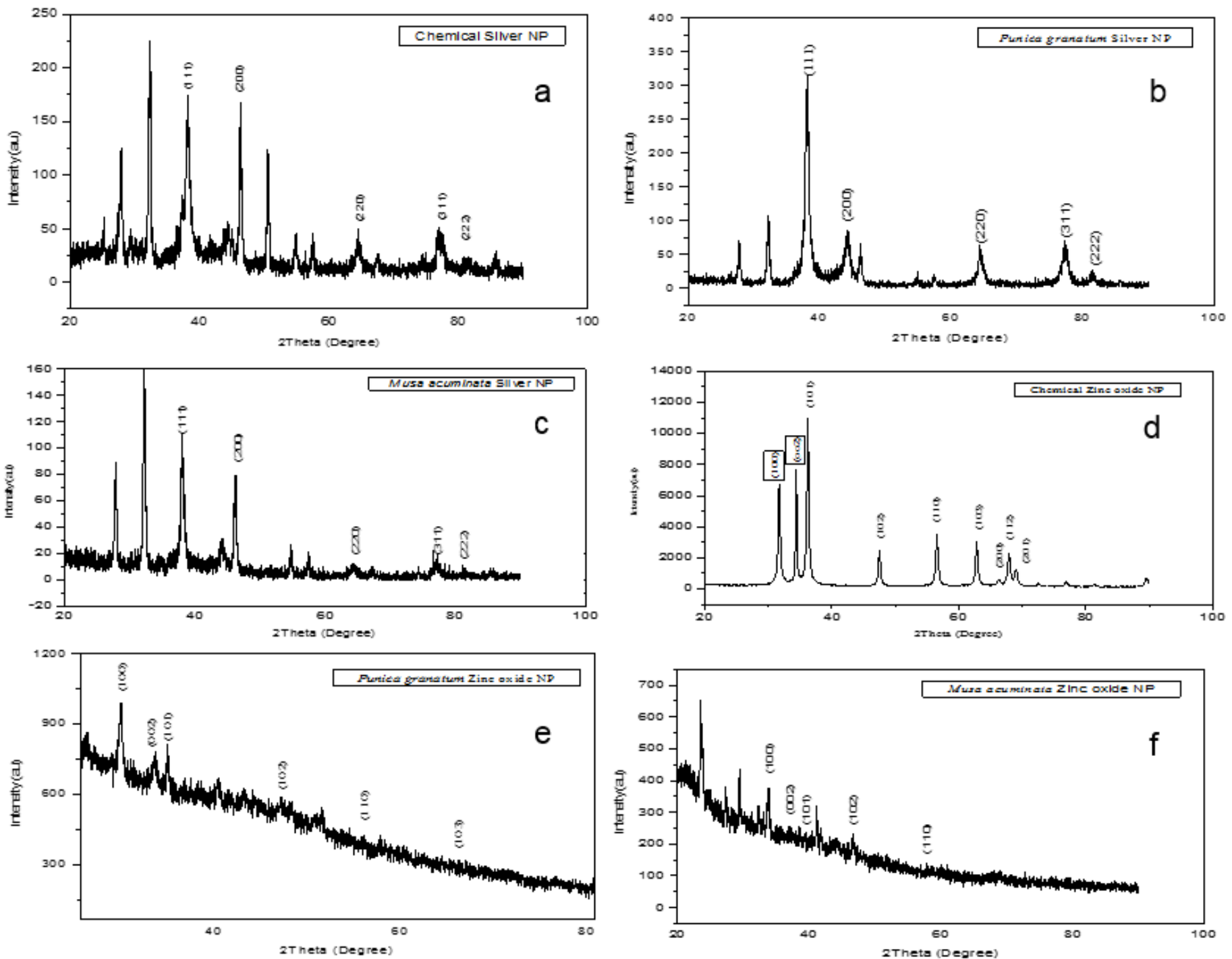

Figure 1

XRD spectra of NPs synthesised by chemical and biological methods (a) Chemically synthesised Ag NPs (b) Ag NPs using P.granatum peels (c) Ag NPs using M. acuminata peels (d) Chemically synthesised ZnO NPs (e) ZnO NPs using P. granatum peels (f) ZnO NPs using M. acuminata peels 

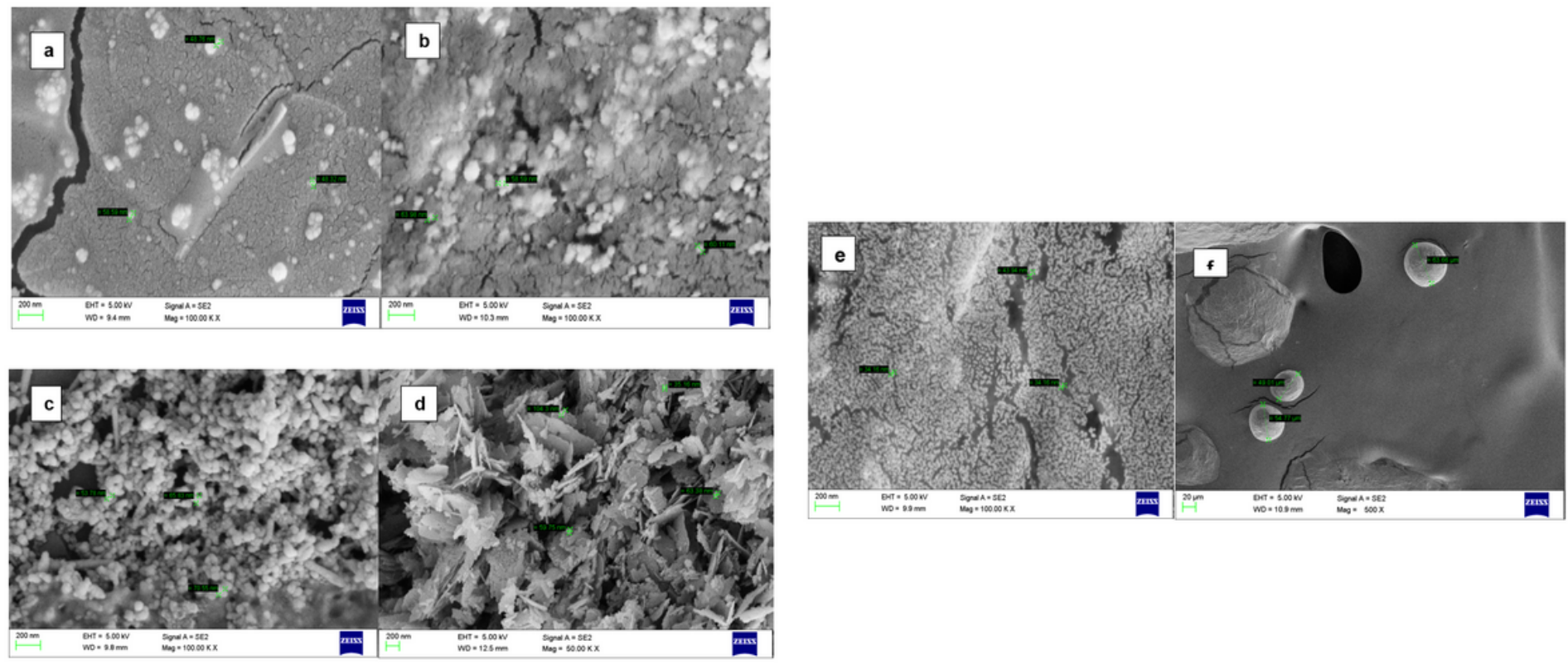

Figure 2

Scanning Electron Micrographs and EDX Profile of the Synthesised NPs (a) SEM image of chemically synthesised Ag NPs (b) SEM image of M. acuminata Ag NPs (c) SEM image of P. granatum Ag NPs (d) SEM image of chemically synthesised ZnO NPs (e) SEM image of M. acuminata ZnO NPs (f) SEM image of P. granatum ZnO NPs

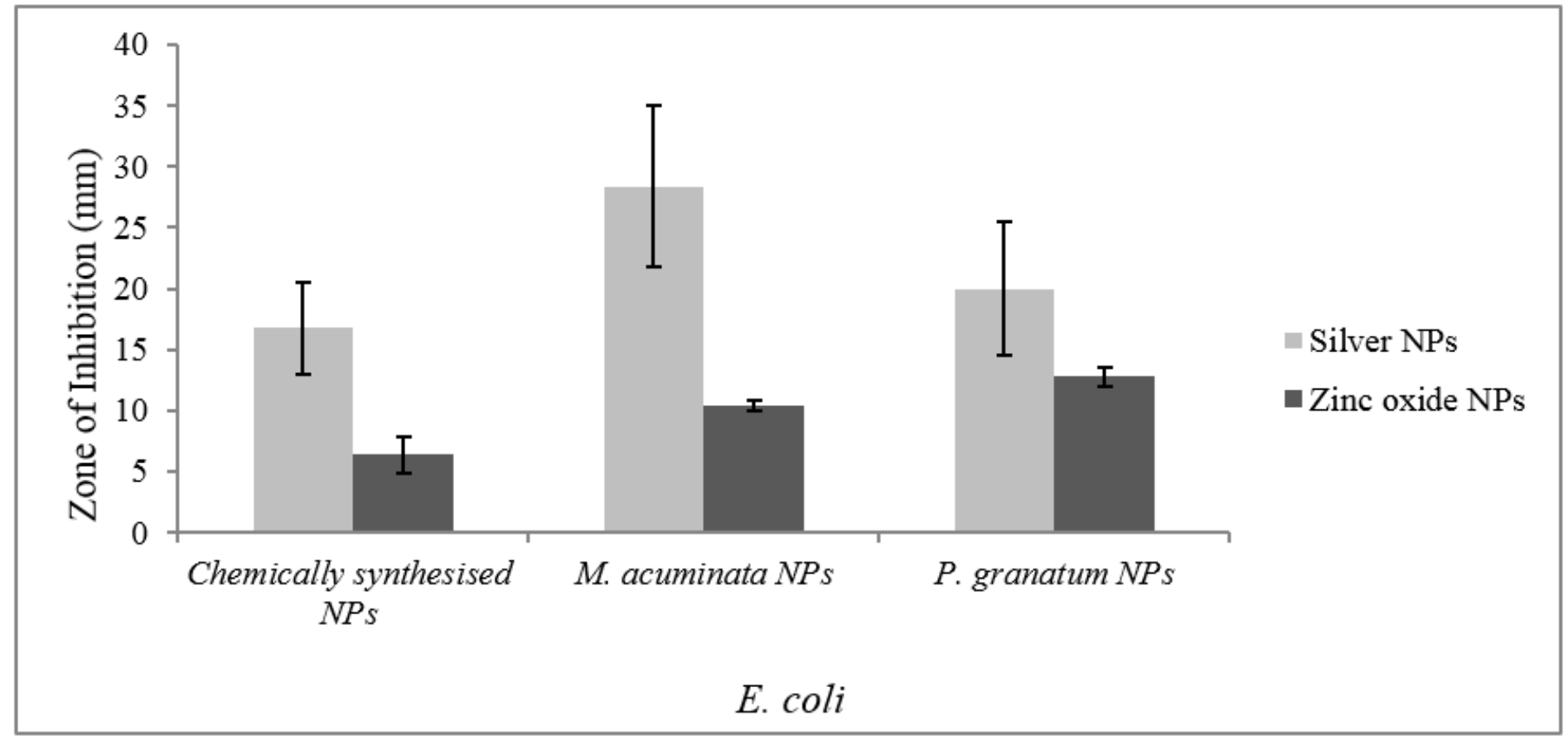

Figure 3

In vitro analysis of the antimicrobial activity of $\mathrm{Ag}$ and $\mathrm{ZnO}$ NPs against $\mathrm{E}$. coli 


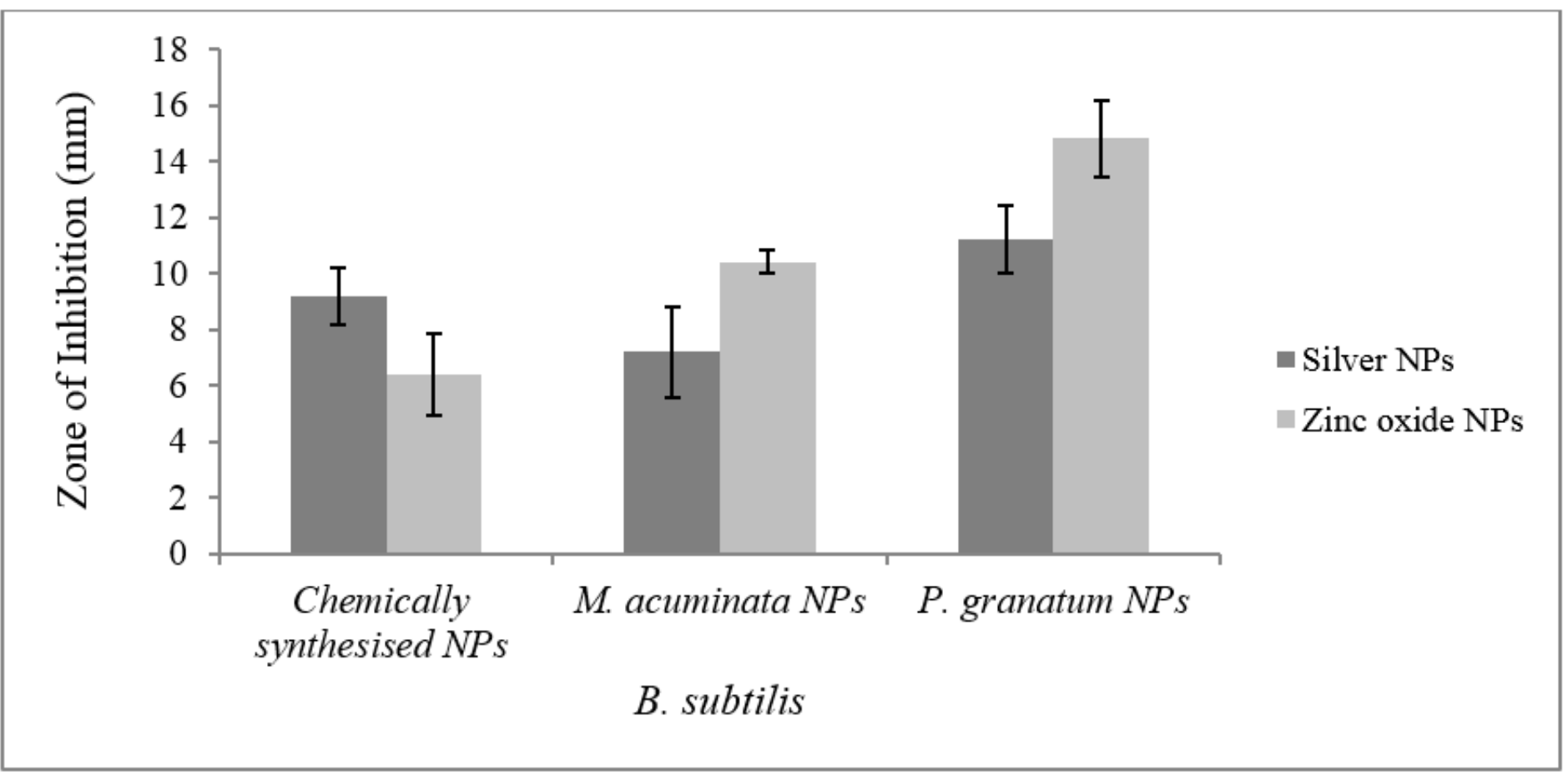

Figure 4

In vitro analysis of the antimicrobial activity of $\mathrm{Ag}$ and $\mathrm{ZnO}$ NPs against $\mathrm{B}$. subtilis. 